\title{
Modeling and use of stellar oscillation visibilities
}

\author{
J. Schou
}

\begin{abstract}
Max-Planck-Institut für Sonnensystemforschung, Justus-von-Liebig-Weg 3, 37077 Göttingen, Germany
e-mail: schou@mps.mpg.de
\end{abstract}

Received 6 February 2017 / Accepted 28 May 2018

\begin{abstract}
Context. Recently our ability to study stars using asteroseismic techniques has increased dramatically, largely through the use of space based photometric observations. Work has also been performed using ground based spectroscopic observations and more is expected in the near future from the SONG network. Unfortunately, the intensity observations have an inferior signal-to-noise ratio and details of the observations do not agree with theory, while the data analysis used in the spectroscopic method has often been based on overly simple models of the spectra.

Aims. The aim is to improve the reliability of measurements of the parameters of stellar oscillations using spectroscopic observations and to enable the optimal use of the observations.

Methods. While previous investigations have used 1D models, I argue that realistic magnetohydrodynamic simulations, combined with radiative transfer calculations, should be used to model the effects of the oscillations on the spectra. I then demonstrate how to calculate the visibility of the oscillation modes for a variety of stellar parameters and fitting methods. In addition to the methods used in previous investigations, I introduce a singular value decomposition based technique. This new technique enables the determination of the information content available from spectral perturbations and allows this content to be expressed most compactly. Finally I describe how the time series obtained may be analyzed.

Results. It is shown that it is important to model the visibilities carefully and that the results deviate substantially from previous models, especially in the presence of rotation. Detailed spectral modeling may be exploited to measure the properties of a larger number of modes than possible via the commonly used cross-correlation method. With moderate rotation, there is as much information in the line shape changes as in the Doppler shift and an outline of how to extract this is given.
\end{abstract}

Key words. asteroseismology - stars: oscillations - techniques: spectroscopic - line: profiles

\section{Introduction}

Most asteroseismic results have so far been obtained from photometric observations using a variety of space-borne instruments, such as WIRE (Fletcher et al. 2006), MOST (Walker et al. 2003), CoRoT (Auvergne et al. 2009), and Kepler (Borucki et al. 2010). This will likely also be the case in the future with the launch of TESS (Ricker et al. 2014) and PLATO (Rauer et al. 2014). The main advantage of photometric observations is that it is possible to observe multiple stars simultaneously, as evidenced by the large number of main sequence stars for which results have been obtained (Stello et al. 2013; Chaplin et al. 2014; Lund et al. 2017a,b). However, Doppler velocity based observations have a superior signal-to-noise ratio. Unfortunately, it is difficult to observe more than one star at a time with a spectrograph based instrument and it is difficult to obtain the required observing time, which is typically weeks to months at a high cadence and high duty cycle on a large telescope; therefore only a few stars have been observed this way, as reviewed by Bedding (2014). With the recent commissioning of the first SONG telescope (Grundahl et al. 2009, 2017) and the planned expansion of the SONG network, more stars will be observed in the future. Given this very limited resource and that the velocity observations are significantly different from photometric observations, it is essential that the maximum information is extracted from the observed stellar spectra and that the resulting power spectra are accurately modeled.
When extracting the oscillation signal from the observed spectra, Doppler shift algorithms developed for radial velocity planet searches (e.g., Bouchy et al. 2001) are often used and it is thus implicitly assumed that the oscillation signal is well represented by a Doppler shift. For fitting power spectra it is generally assumed that mode visibilities, as a function of the azimuthal order $m$, follow the description of Gizon $\&$ Solanki (2003), where it was assumed that the sensitivity only depends on the center-tolimb distance on the stellar disk. Below I will show that, in the presence of modest stellar rotation, the Doppler shift approach discards a significant amount of information and that the use of the visibility expression of Gizon \& Solanki (2003) can lead to significant systematic errors. The dependence of the visibilities on the spherical harmonic degree $l$ is typically kept free or prescribed to follow a relationship based on simplified models, even though it is known that the observed visibilities for the Sun do not agree with the theoretically expected values for broadband photometry (Lund et al. 2014).

A few results, similar to those presented in the present paper, but using a different set of simulations were shown in Schou (2014). The present paper expands substantially on this by considering stars of different spectral types, the effects of magnetic fields, and a variety of analysis methods.

Models of the effects of oscillations on spectra and how the information can be extracted have been studied in the past; see for example Brown et al. (1998), Briquet \& Aerts (2003), and 
particularly Zima (2006) in which several physical effects were included. However, these models have used simplified line models, rather than the results of 3D magnetohydrodynamic (MHD) models, such as those used in the present work and the effects of magnetic fields on the spectra were not considered. The use of a singular value decomposition (SVD) based analysis was also not discussed in those studies. Rather, Briquet \& Aerts (2003), for example, used a moments based method, which does not result in as compact a representation as the SVD based method.

In Sect. 2, I start by discussing details of the models, the radiative transfer, and how the spectral perturbations can be calculated. In Sect. 3, I consider a variety of analysis methods, starting with the classic cross-correlation. As that is shown to be suboptimal, I also discuss the option of performing least-squares fits and describe an SVD based method. As these methods result in multiple time series, I finish Sect. 3 by outlining how multiple time series can be analyzed. Finally I discuss some of the issues still to be addressed and conclude.

Issues of how the methods may be applied to a given spectrograph, how they may be made more numerically efficient, and the analysis of simulated or real time series are deferred for later consideration.

\section{Background}

Before addressing the fitting of the spectra I will discuss, in the following subsections, the MHD models used, the radiative transfer performed, and how those are used to derive the perturbations to the spectra.

\subsection{Convection models and radiative transfer}

The convection models used in this work are snapshots from the simulations of Beeck et al. (2013) with surface properties corresponding to main sequence stellar models of types $\mathrm{F} 3, \mathrm{G} 2, \mathrm{~K} 0, \mathrm{~K} 5, \mathrm{M} 0$, and $\mathrm{M} 2$ and with sizes ranging from $30 \mathrm{Mm} \times 30 \mathrm{Mm} \times 9 \mathrm{Mm}$ for the $\mathrm{F} 3$ model to $1.56 \mathrm{Mm} \times 1.56 \mathrm{Mm} \times 0.8 \mathrm{Mm}$ for the $\mathrm{M} 2$ model. For each spectral type, models with injected magnetic fields of $0 \mathrm{G}, 20 \mathrm{G}$, $100 \mathrm{G}$, and $500 \mathrm{G}$ were made. Beeck et al. (2013) provide further details of the various simulations. For the radiative transfer, SPINOR (Frutiger et al. 2000) was used to synthesize various lines for a selection of the models discussed above.

The main line studied is FeI at $6173 \AA$, which is used by the HMI instrument (Schou et al. 2012) and has the advantage that it has simple atomic properties, a large Landé Factor, is relatively free of blends and that some of the results can be verified by observations. For selected models the FeI line at $5250 \AA$ and the SiI line at $10827 \AA$ were also used. In addition to these real lines, the $6173 \AA$ line was also modeled, assuming abundances of 0.1 and 10 times solar, to simulate weaker and stronger lines at otherwise fixed atomic physics. Finally a line synthesis (" $500 \mathrm{H}$ ") is performed for the $500 \mathrm{G}$ case with the magnetic field artificially set to zero. This makes it possible to disentangle the effects of the field on the MHD simulations and on the final radiative transfer calculation.

The line profiles are synthesized for a number of viewing angles at a spectral resolution of $7.5 \mathrm{~m} \AA$ with 201 points covering $\pm 0.75 \AA$ (except for the SiI $10827 \AA$ line, where the spacing is $30 \mathrm{~m} \AA$ covering $\pm 3 \AA$ in order to accommodate the wider line) and the resulting profiles are averaged horizontally.

Figure 1 shows the profiles for the $\mathrm{G} 2$ star at $0 \mathrm{G}$. The limb darkening is clearly visible as a decrease of the continuum with

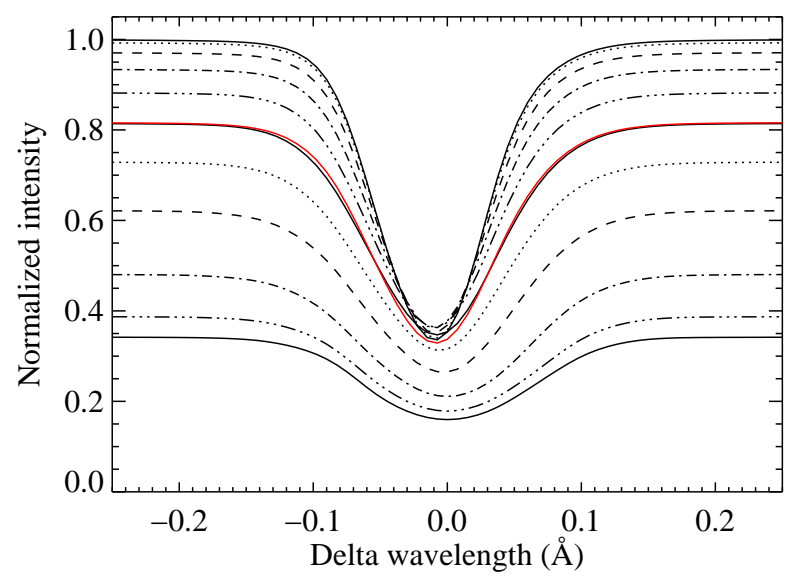

Fig. 1. Line profiles for the FeI $6173 \AA$ line for the $\mathrm{G} 2$ star with $0 \mathrm{G}$. From top to bottom the viewing angles are $0^{\circ}, 10^{\circ}, \ldots, 80^{\circ}, 85^{\circ}$, and $87^{\circ}$. The profiles were normalized to the disk center continuum. The red line indicates the disk averaged line profile in the absence of rotation.

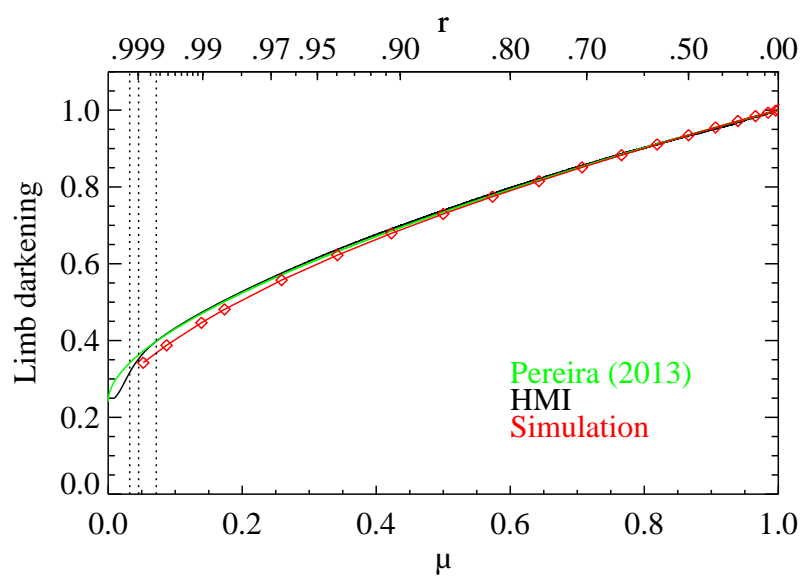

Fig. 2. Continuum limb darkening near the FeI line at $6173 \AA$ A. Results are shown both as a function of the fractional solar radius $r$ (on the top axis) and $\mu=\sqrt{1-r^{2}}$ (on the bottom axis). Red: From the simulations shown in Fig. 1, including a number of additional angles. Black: An estimate from the HMI instrument. Green: An estimate from Pereira et al. (2013) courtesy of R. Trampedach. Vertical dotted lines indicate 1.0, 2.0, and 5.0 pixels from the limb. The results were not corrected for the PSF of the HMI instrument.

viewing angle, as is the broadening of the line toward the limb. The line also becomes shallower and shifts to the red toward the limb because of convective blueshift. Of these quantities the limb darkening is the easiest to verify observationally and Fig. 2 shows a comparison between the observed limb darkening from HMI and that from various calculations. In general the correspondence is good, but there are also differences, especially very close to the limb where the point spread function (PSF) of HMI has not been taken into account. More importantly, the calculated limb darkening appears to be too steep, even far from the limb. Interestingly, the results of Pereira et al. (2013) are significantly closer to the observed results. These authors concluded that the key improvement in their calculations is that the radiative transfer within their MHD simulation was performed more accurately, resulting in structural changes near the surface. Such detailed radiative transfer is typically not performed as part of large MHD simulations due to computational cost, but was carried out by Pereira et al. (2013) to better model the center-to-limb varia- 
tions of various observed quantities. The radiative transfer used for the detailed line synthesis, given an MHD cube, is believed to be accurate for both their computations and those performed in the present study.

In any case, the limb darkening is well modeled, giving confidence in the accuracy of the simulations and radiative transfer. For other stars Dravins et al. (2017), recently demonstrated that the accuracy of the line profile calculations may be checked by using spectroscopic observations during planetary transits.

\subsection{Effects of oscillations on spectra}

To calculate the effect of the oscillations on the observed spectra, it is necessary to integrate the oscillations over the stellar disk $(\odot)$ as follows:

$$
\begin{aligned}
I(\lambda, t) & =\int_{\odot} I(x, y, \lambda, t) \mathrm{d} \boldsymbol{r} \\
& =\int_{\odot} I_{\mathrm{m}}\left(\mu, \lambda+k V_{0}+k V^{\prime}(t)\right) \mathrm{d} \boldsymbol{r} \\
& \approx \int_{\odot} I_{\mathrm{m}}\left(\mu, \lambda+k V_{0}\right) \mathrm{d} \boldsymbol{r}+\int_{\odot} k V^{\prime}(t) I_{\mathrm{m}}^{\prime}\left(\mu, \lambda+k V_{0}\right) \mathrm{d} \boldsymbol{r} \\
& =I_{0}(\lambda)+k \int_{\odot} V^{\prime}(t) I_{\mathrm{m}}^{\prime}\left(\mu, \lambda+k V_{0}\right) \mathrm{d} \boldsymbol{r} \\
& \equiv I_{0}(\lambda)+\delta I(\lambda, t),
\end{aligned}
$$

where $\lambda$ is the wavelength, $t$ time, $x$ and $y$ are the coordinates relative to disk center (in units of the stellar radius), $\boldsymbol{r}=(x, y)$, $\mu^{2}=1-x^{2}-y^{2}=1-r^{2}, r$ is the fractional radius, $I_{\mathrm{m}}$ the model intensity, $I_{\mathrm{m}}^{\prime}=\mathrm{d} I_{\mathrm{m}} / \mathrm{d} \lambda, V_{0}$ the background stellar LOS velocity (assumed in this work to be due to solid body rotation), $V^{\prime}$ is the LOS velocity induced by the mode (assumed small), $k=d \lambda / d V=\lambda / c$, and $c$ is the speed of light. For simplicity the dependencies of $V_{0}$ and $V^{\prime}$ on $x$ and $y$ and the fact that $\lambda$ is in reality discretized are suppressed here and in the rest of this article. Similarly time variations of $V_{0}$ have been ignored. An obvious source of such variations is the orbital motion of the Earth. This causes variations in $V_{0}$, which are far outside of the linear range and which have to be dealt with by shifting the spectra or fitting templates. It has been assumed that the only change to the spectrum, at a given spatial location, caused by the mode is that resulting from Doppler shift. In other words intensity, linewidth, and other thermodynamic changes are not taken into account.

To calculate the effect of a given mode, it is necessary to calculate the LOS velocity caused by it. Assuming that the modes have unit amplitude, are undistorted by asphericities (such as rotation or starspots), are undamped, and that only the radial component is significant, the induced velocity is given by

$$
\begin{aligned}
V^{\prime}(x, y, t) & =\mathfrak{R}\left(\mu Y_{l}^{m}(x, y) \mathrm{e}^{-\mathrm{i} \omega_{l m} \mathrm{t}}\right) \\
& =\mathfrak{R}\left(\mu P_{l}^{|m|}(\sin \theta) \mathrm{e}^{\mathrm{im} \phi-\mathrm{i} \omega_{l} \mathrm{mt}}\right) \\
& =\mu P_{l}^{|m|}(\sin \theta)\left(\cos \omega_{l m} t \cos m \phi+\sin \omega_{l m} t \sin m \phi\right) \\
& \equiv V_{l m}^{c} \cos \omega_{l m} t+V_{l m}^{s} \sin \omega_{l m} t
\end{aligned}
$$

where the factor $\mu$ accounts for the velocity projection factor, $Y_{l}^{m}$ is a spherical harmonic, $P_{l}^{m}$ an associated Legendre function, $x=\sin \phi \cos \theta, y=\sin \theta \sin i-\cos \phi \cos \theta \cos i, \phi$ is longitude, $\theta$ is latitude, $i$ is the inclination of the rotation axis, and $\omega_{l m}$ is the mode frequency. With the convention chosen $V_{l-m}^{c}=V_{l m}^{c}$ and $V_{l-m}^{s}=-V_{l m}^{s}$. Without loss of generality the coordinate system was chosen to have the stellar rotation axis aligned with the $y$ axis.
Substituting Eq. (2) into Eq. (1),

$$
\begin{aligned}
\delta I(\lambda, t) & =k \int_{\odot}\left(V_{l m}^{c} \cos \omega t+V_{l m}^{s} \sin \omega t\right) I_{\mathrm{m}}^{\prime}\left(\mu, \lambda+k V_{0}\right) \mathrm{d} \boldsymbol{r} \\
& \equiv \delta I_{l m}^{c}(\lambda) \cos \omega t+\delta I_{l m}^{s}(\lambda) \sin \omega t
\end{aligned}
$$

is obtained. It is important to note that $\delta I_{l-m}^{c}=\delta I_{l m}^{c}$ and $\delta I_{l-m}^{s}=$ $-\delta I_{l m}^{s}$ and that in the absence of E-W asymmetries (e.g., rotation) the $\delta I_{I m}^{s}$ term vanishes.

While I have, for simplicity, assumed that the only background velocity is that from uniform rotation and that the modes are undistorted, the formalism can easily accommodate the more general case. For some details of how this can be carried out, see Zima (2006). Similarly it is straightforward to include thermodynamic changes, when known.

\section{Fitting methods}

In this section I describe the results of three methods for extracting information from the observed spectra: a simple crosscorrelation, a fit of the expected perturbations, and an SVD based analysis.

\subsection{Cross-correlation analysis}

A simple and common method used to extract the Doppler shift is to cross-correlate the reference spectrum with the observed spectrum, as described by, for example, Bouchy et al. (2001). In principle the wavelength dependence of the noise should be taken into account, but for simplicity I start by assuming a uniform noise and discuss the effect of the wavelength dependence of the noise in the next subsection.

As the oscillation velocities are small and do not cause a significant smearing of the line (relative to the zero oscillation case) the reference spectrum can be taken as $I_{0}$. Given that the perturbations are small, the cross-correlation is equivalent to a fit of $\delta I$ at each time, to the derivative $I_{0}^{\prime}$ of $I_{0}$ with respect to $\lambda$, assuming a uniform error, using the following equation:

$\delta I(\lambda, t)=V_{\text {fit }}(t) k I_{0}^{\prime}(\lambda)$,

where $V_{\text {fit }}$ is the fitted velocity. Given Eq. (3), and since a unit amplitude oscillation was assumed, the visibilities, $S$, can be defined by fitting the perturbations to the derivative, that is,

$\delta I_{l m}^{c}(\lambda)=S_{l m}^{c} k I_{0}^{\prime}(\lambda)$

and

$\delta I_{l m}^{s}(\lambda)=S_{l m}^{s} k I_{0}^{\prime}(\lambda)$

As discussed later, only $S_{l m}^{c}$ is significant.

To illustrate how well these profiles match, Fig. 3 shows $\delta I_{l m}^{c}$ for various modes together with fits to $I_{0}^{\prime}$. For the lowest degrees the fit of $I_{0}^{\prime}$ is very good but it starts to disagree more as the degree is increased. Still, the approximation used for Eq. (4) is perhaps adequate to justify using the cross-correlation method for this case. The decrease of the visibility at higher $l$ is also evident.

If the star is rotating and not observed from the pole $\left(i=0^{\circ}\right)$ the results change substantially, as illustrated in Fig. 4. While $\delta I_{l m}^{c}$ is still moderately well fitted by $I_{0}^{\prime}, \delta I_{l m}^{s}$ is now nonzero and shows a very different profile, corresponding to a narrowing and widening of the line, which is not well fitted by the derivative. I return to this issue below. 


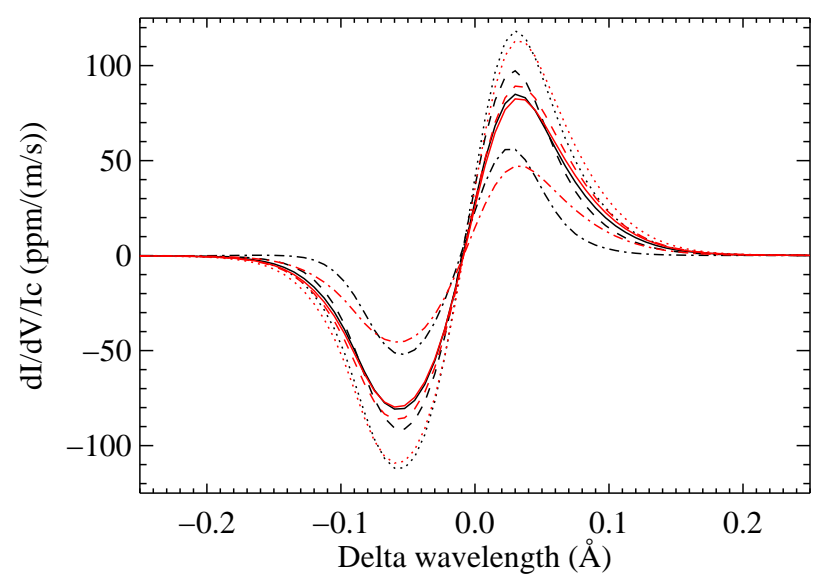

Fig. 3. Perturbations $\delta I_{l m}^{c}$ (black) for the $6173 \AA$ line for the $\mathrm{G} 2$ star with $0 G, i=0^{\circ}$ and no rotation. The values of $(l, m)$ are $(0,0)$ (solid), $(1,0)$ (dotted), $(2,0)$ (dashed), and $(3,0)$ (dash-dotted). Also shown (in red) is the derivative $I_{0}^{\prime}$ scaled to best fit each perturbation. The shape (but not magnitude) of the perturbations do not depend on $i$ and $m$ in the absence of rotation.

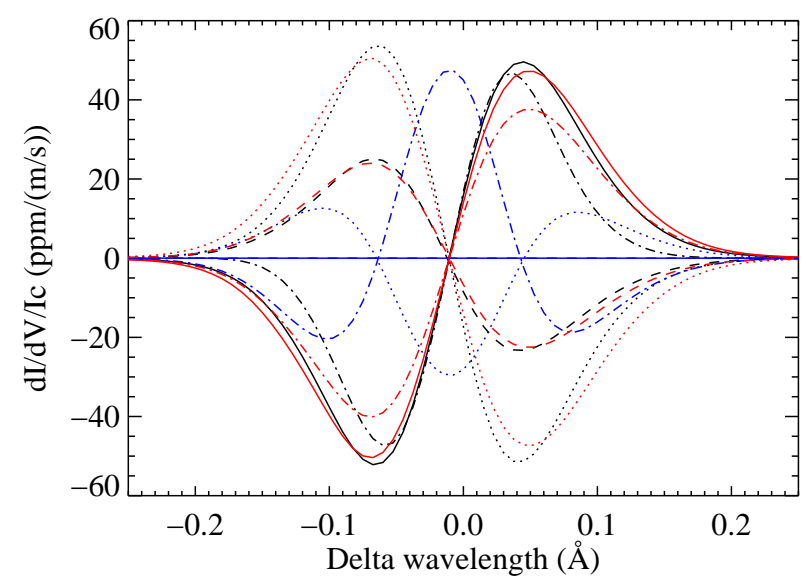

Fig. 4. Similar to Fig. 3 this shows the perturbations $\delta I_{l m}^{c}$ (black) and $\delta I_{l m}^{s}$ (blue) for the $\mathrm{G} 2$ star with $i=90^{\circ}$ and solid body rotation with an equatorial rotation velocity of $4 \mathrm{~km} \mathrm{~s}^{-1}$. The values of $(l, m)$ are $(0,0)$ (solid), $(1,1)$ (dotted), $(2,0)$ (dashed), and $(2,2)$ (dash-dotted). Also shown (in red) is the derivative $I_{0}^{\prime}$ scaled to best fit each of the $\delta I_{l m}^{c}$. The unperturbed line, and thus the derivative, is different from that in Fig. 3 because of rotational broadening.

Examples of the visibilities $S_{l m}^{c}$ are shown in Fig. 5 for a variety of spectral lines. As can be seen the differences are modest, especially in the visible, even if the abundances are changed substantially. The SiI line shows a slightly different behavior, likely because it is a much broader line and in the near-infrared. As such it should be possible to model only selected lines and interpolate the results. For different stars (Fig. 6) there is a modest trend with stellar type, but the overall trend remains the same. Again it would appear that while the variations should not be neglected, it should be possible to interpolate between the spectral types, avoiding the need to run large simulations for each star.

Adding a modest magnetic field also does not have a dramatic effect as shown in Fig. 7. For the, possibly unrealistic, $500 \mathrm{G}$ case the change is more significant. It is interesting that the changes in the structure dominates over the radiative transfer, as can be seen by turning off the field in the $500 \mathrm{H}$ case, which changes the result by much less than the introduction of the

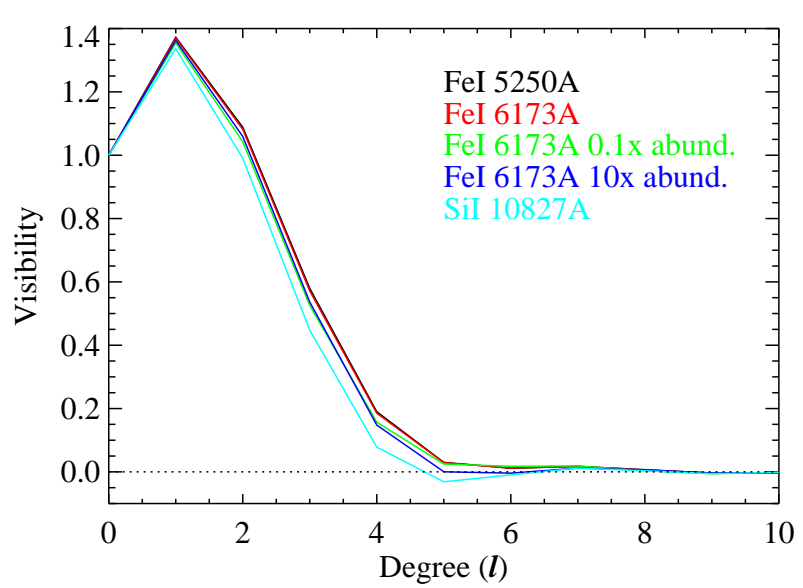

Fig. 5. Visibilities for the $m=0$ modes for the $\mathrm{G} 2$ star as a function of $l$ and spectral line, for the $0 G$ case and an inclination angle of $0^{\circ}$. In addition to the regular lines, profiles were synthesized with larger and smaller abundances of Fe for the $6173 \AA$ line (using the same simulations). For clarity the visibilities have, for each spectral line, been divided by the visibility of $l=0$. The visibilities of the other $m$ values are zero at $i=0^{\circ}$.

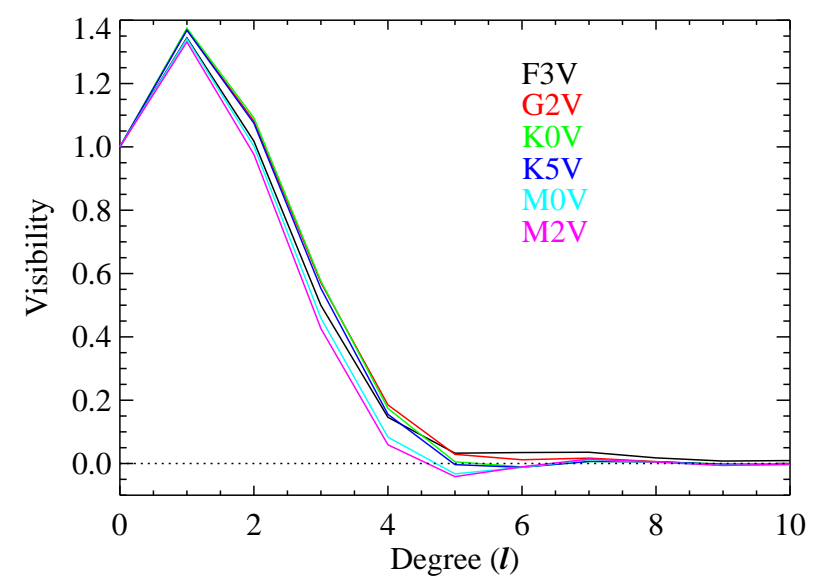

Fig. 6. Similar to Fig. 5 for different stars. All cases are for the $6173 \AA$ line without a magnetic field, no rotation, $i=0^{\circ}$, and $m=0$, and the visibilities have been divided by that for $m=l=0$.

magnetic field in the MHD simulations. As such it appears that the presence of magnetic fields can probably be ignored unless the fields are very strong.

The inclination dependence of the visibilities shown in Fig. 8 is much more interesting. Without rotation the inclination dependence follows the prediction by (Gizon \& Solanki 2003; not shown). But even for a modest rotation rate of $4 \mathrm{~km} \mathrm{~s}^{-1}$ (roughly twice solar) the deviations are dramatic. For an equatorial observation $\left(i=90^{\circ}\right)$ the ratio of the visibility of the $(l, m)=(3,3)$ mode relative to that of the $(3,1)$ mode changes from -1.29 in the absence of rotation to -2.71 .

That the rotation has a significant effect on the visibilities is is not too surprising. As the rotation increases, the line emitted far from the central meridian moves far away from that at the center (and the average) and so the measurement ceases to be sensitive to the oscillations near the edges. This also explains why the changes become significant when the Doppler shift at the east and west limbs becomes comparable to the linewidth; the full width half maximum (FWHM) of the average $6173 \AA$ line in the nonrotating case corresponds to about $5.3 \mathrm{~km} \mathrm{~s}^{-1}$. 


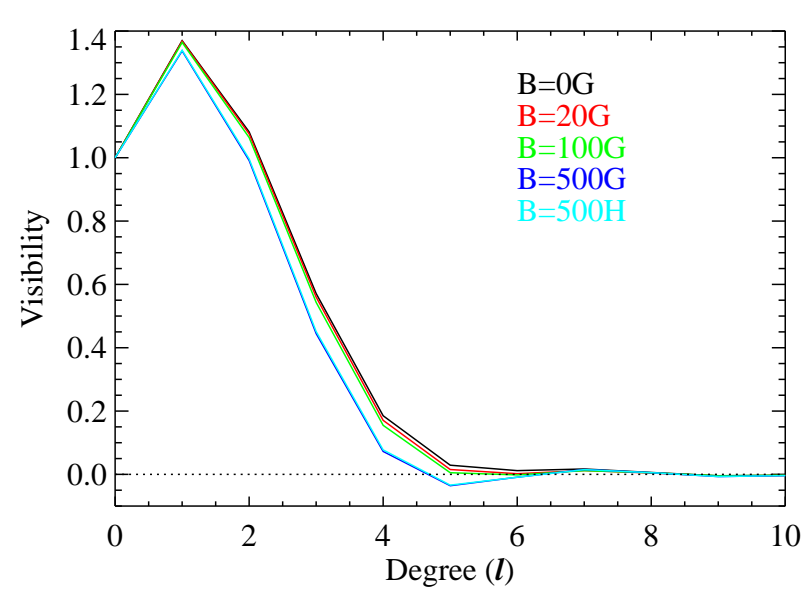

Fig. 7. Similar to Fig. 5 for the different field strength cases in Beeck et al. (2013). The " $500 \mathrm{H}$ " case used the " $500 \mathrm{G}$ " simulation but with the field set to zero in the radiative transfer calculations. All cases are for the G2 star using the $6173 \AA$ line, no rotation, $i=0^{\circ}$ and $m=0$ and the visibilities have been divided by the one for $m=l=0$.

This issue is illustrated in Fig. 9, in which the sensitivity as a function of position on the stellar disk is shown for an edge-on $\left(i=90^{\circ}\right)$ view. As the rotation rate increases the sensitivity becomes more and more concentrated toward the central meridian. Beyond about $4 \mathrm{~km} \mathrm{~s}^{-1}$ the sensitivity becomes negative at the east and west limbs as the shift becomes comparable to the linewidth. While barely visible it is also the case that the sensitivity is not exactly east-west symmetric as a consequence of the combination of the rotation and convective blueshift.

In solar-like stars the spacing between modes with different $m$ (which is essentially equal to the rotation frequency) is often small or comparable to the linewidth (Bazot et al. 2007; Nielsen et al. 2014), resulting in the modes blending together. In this case, it is therefore necessary to rely on a model of the visibilities and any errors in that can lead to incorrect mode parameter estimates. This is illustrated in Fig. 10. In this figure, limit spectra calculated with the correct visibilities (those including the effects of rotation) were fitted assuming the incorrect visibilities (those not including the effects of rotation). As is shown, both the inclinations and splittings, that is, the spacing between adjacent $m$ values, which is essentially equal to the rotation frequency for Sun-like stars, are misestimated; in some cases this results in values far from the input values. Also, the values determined from different values of $l$ are inconsistent, which may or may not be noticeable. When the peaks corresponding to the different values of $m$ are well separated, the error is obvious and would make it clear that there is a problem. If the correct visibilities are used, the parameters are, of course, correctly estimated. On the other hand the errors are suboptimal, as discussed in Sect. 3.3.

Taking into account the effects of rotation does not mean that it is necessary to fit for additional parameters. The visibilities are given by the rotation rate (and thus the splittings) and inclination, both of which are already fitted for. The only required change to a fitting code is thus to replace the use of the expressions in Gizon \& Solanki (2003) by a parametrization of the visibilities (e.g., the results shown in Fig. 8), for example by interpolating the values.

\subsection{Least-squares fit}

As mentioned in the previous subsection, the cross-correlation analysis suffers from several problems. Among others, the effects of rotation can be substantial and the implicit assumption that the derivative of the line is a good approximation to the perturbation is thus poor. Also, it is assumed that the noise is independent of wavelength. These approximations can be addressed by performing a maximum-likelihood fit, which is considered in this subsection.

That the noise is independent of the wavelength is unlikely to be correct, as discussed in Bouchy et al. (2001). A better approximation is to assume that photon noise is the dominant noise term. Assuming that the number of photons is $>>1$ and that the perturbation is small, the photon noise can be approximated by a normal distribution with a variance proportional to the signal. A fairly benign consequence of this is that an internal estimate of the noise on the derived velocity, assuming that the noise equals that in the continuum, is wrong relative to that using the photon noise estimate. In the case of the $6173 \AA$ line by an $l$-independent factor around 1.2.

A more significant problem is that the estimate is statistically suboptimal, in other words that an estimate with a better signalto-noise ratio can be constructed. The optimal (maximumlikelihood) estimate can be obtained by performing an error weighted least-squares fit, writing the observed spectrum as

$I(\lambda, t)=I_{0}(\lambda)+x_{\mathrm{c}}(t) \delta I_{l m}^{c}(\lambda)+x_{\mathrm{s}}(t) \delta I_{l m}^{s}(\lambda)$,

thereby obtaining a time series $x_{\mathrm{c}}$ of the cosine component and another $x_{\mathrm{s}}$ of the sine component.

The error weighted fit is equivalent to an unweighted fit in which both the data and model are divided by the error estimate, which is in this case given by the square root of the model. A downside of the least-squares fitting is that a different function should ideally be fitted for each mode. This is discussed in the next subsection.

A comparison between the cross-correlation estimates and the fits of only the cosine component (which is dominant at low rotational velocity) is shown in Fig. 11. In this figure, the signalto-noise ratio is shown, rather than the visibility. As the noise is independent of the mode in the cross-correlation case, this is proportional to the absolute value of the visibility. For the polar $\left(i=0^{\circ}\right)$ case, which is unaffected by rotation, the effect is modest. But for an equatorial $\left(i=90^{\circ}\right)$ view the difference is significant, especially at higher degree. At $l=4$ the improvement is roughly a factor of 1.6 and at $l=5$ it is 2.2 .

Results of fitting for both the sine and cosine components are shown in Fig. 12. At modest rotation rates the visibility of the real part drops and the visibility of the imaginary part increases. At higher degrees the two visibilities become similar. In other words the broadening and narrowing of the line caused by the sin component becomes as significant as the shift of the line caused by the cos component, which among other things allows for separating prograde and retrograde modes. In general the ability to observe two separate time series opens up the possibility of deriving significantly more information about the modes, but requires a somewhat more complex analysis, as described in Sect. 3.4.

While the least-squares fitting does provide significant advantages over the cross-correlation approach, the SVD based approached, discussed in the next subsection, provides significant advantages, and so the practicalities of implementing the least-squares fitting are not discussed further.

\subsection{SVD analysis}

The fact that the perturbations caused by different modes are different raises a number of questions. Is it necessary to fit a 

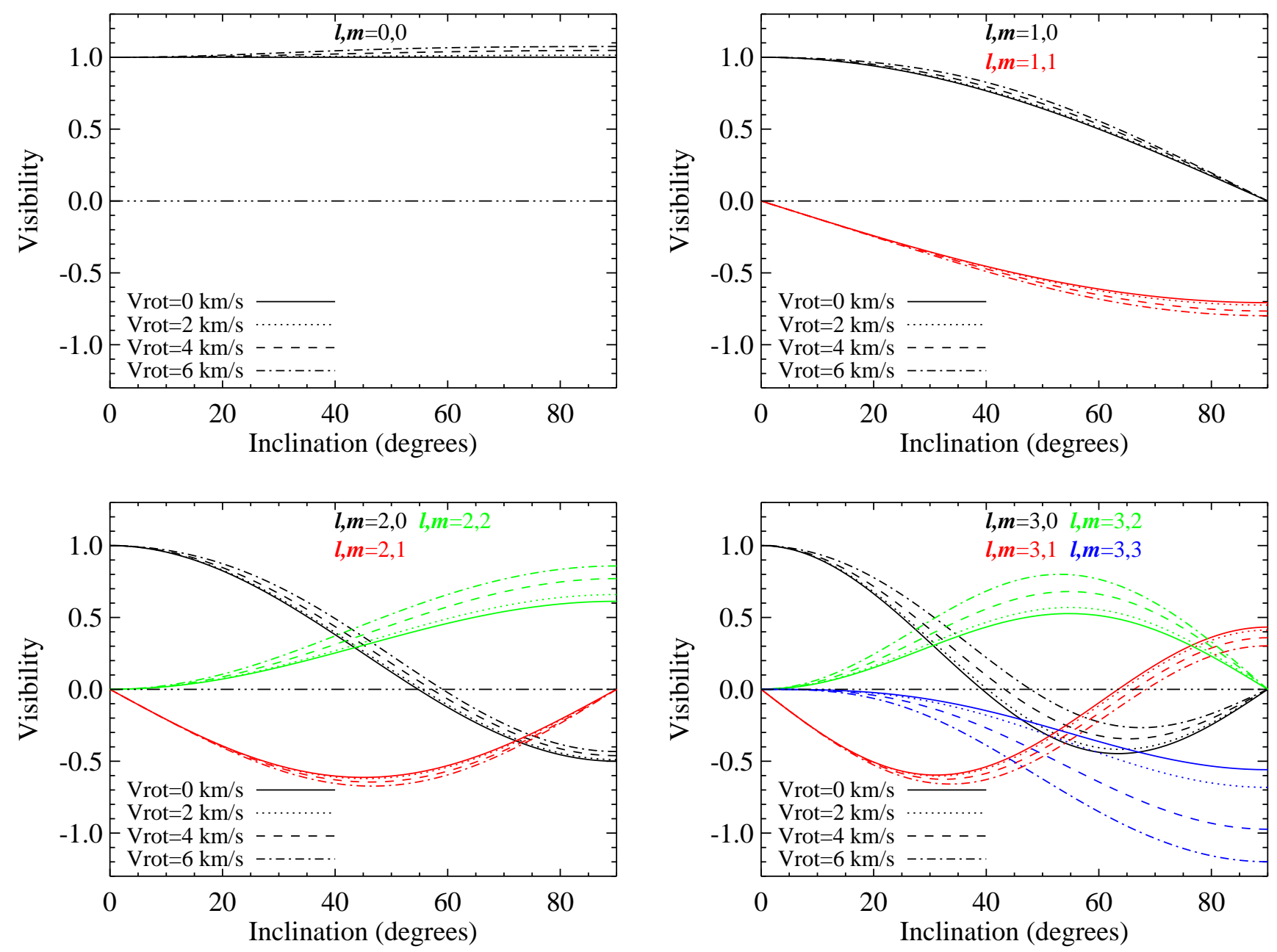

Fig. 8. Visibilities $S_{l m}^{c}$ of the $l=0$ through $l=3$ modes for the G2 star as a function of inclination angle and equatorial rotation velocity for the case with $0 G$ and using the $6173 \AA$ Aine. For clarity the values have been divided by the visibility of the corresponding $m=0$ mode at $i=0^{\circ}$. The corresponding $S_{l m}^{s}$ values, which are not shown, go up to 0.02 , but are generally less than 0.01 .

given spectrum multiple times with functions designed to fit each mode? Is it possible to separate more than the cos and sin components, like different $l$ and $m$ values? A way to address these questions is to perform an SVD (Principal Component) analysis, writing the perturbations as

$$
\frac{\delta I_{j}(\lambda)}{\sqrt{I_{0}(\lambda)}}=\sum_{k} \boldsymbol{U}_{k, j} \sigma_{k} \boldsymbol{V}_{k}(\lambda),
$$

where the division by $\sqrt{I_{0}(\lambda)}$ accounts for the variation of the photon noise, $j$ encodes the mode ID, $\sigma_{k}$ are the singular values, $\boldsymbol{U}$ the singular vectors in mode ID, and $\boldsymbol{V}$ are the singular vectors in wavelength. The mode ID could, for example, be $j=(l, m, p)$, where $p$ is $c$ or $s$ as defined in Eq. (3) at some fixed inclination and rotation rate. The properties of the SVD ensure that the $\boldsymbol{U}$ vectors are orthonormal, as are the $\boldsymbol{V}$ vectors. By convention, the singular values $\sigma$ are ordered by descending value. Their squares give the amount of variance of the left-hand side of Eq. (8) that is captured by the corresponding term (having implicitly assumed that the inherent mode amplitudes are independent $l$ and $m$, as expected for solar-like oscillations on a Sun-like star). An important property of the SVD is that the variance captured by the first $N$ terms is the maximum possible. No other vectors, such as the moments used by Briquet \& Aerts (2003), can capture more information using $N$ terms.
The vectors $\boldsymbol{V}$ are fitted to the spectra using an unweighted least-squares fit of

$$
\frac{I(\lambda, t)-I_{0}(\lambda)}{\sqrt{I_{0}(\lambda)}}=\sum_{k=1}^{k_{\max }} y_{k}(t) \boldsymbol{V}_{k}(\lambda),
$$

for some suitable $k_{\max }$ (see discussion later in this subsection), resulting in several time series $y_{k}$. As the $\boldsymbol{V}$ vectors are orthonormal, the noise on each term is the same and the $y_{k}$ are given by dot products

$y_{k}(t)=\sum_{\lambda} V_{k}(\lambda) \frac{I(\lambda, t)-I_{0}(\lambda)}{\sqrt{I_{0}(\lambda)}}$,

in the case where the fits are performed over the same wavelengths as the SVD. It also follows that the $\sigma U$ are the corresponding visibilities of the various modes.

Figure 13 shows the singular vectors in wavelength for three different cases and as can be seen the vectors are very similar. As expected given the earlier results, the vectors look roughly like the first, second, and third derivatives of the spectral line with wavelength.

On the other hand the singular values $\sigma$ are somewhat different for the different cases. A way to quantify this is to ask how much of the variance (i.e., information in the spectra) is 


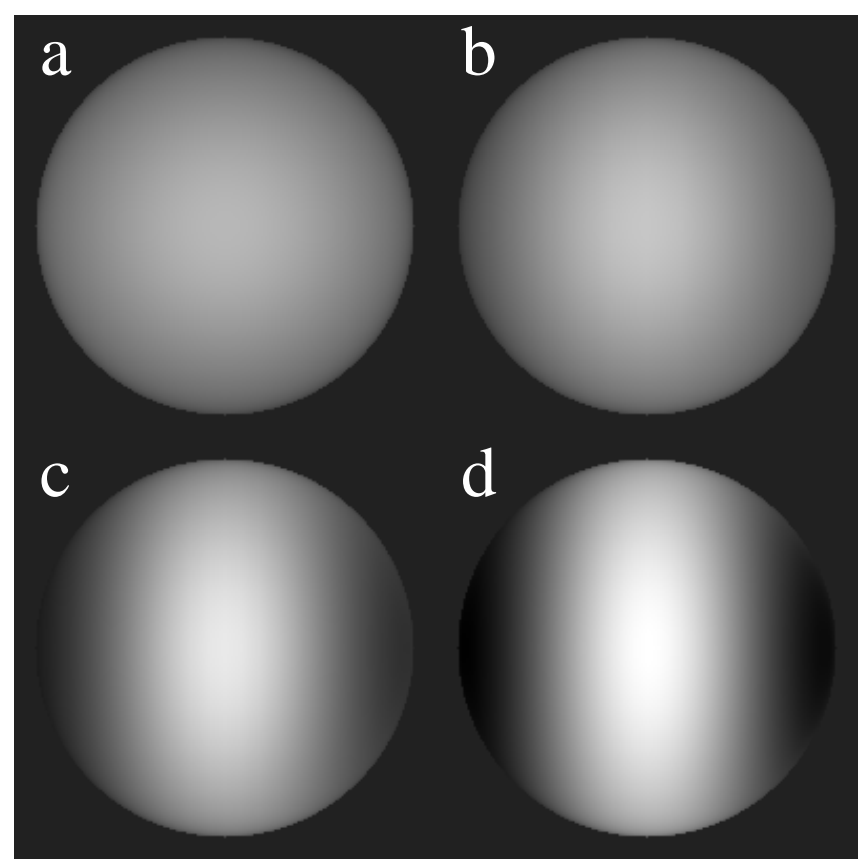

Fig. 9. Sensitivity as a function of position on the disk for the $6173 \AA$ line for the $\mathrm{G} 2$ star with $0 G$ and $i=90^{\circ}$. The rotation rates for panels a through $d$ are $0 \mathrm{~km} \mathrm{~s}^{-1}, 2 \mathrm{~km} \mathrm{~s}^{-1}, 4 \mathrm{~km} \mathrm{~s}^{-1}$, and $6 \mathrm{~km} \mathrm{~s}^{-1}$. The maps are normalized to have the same integrated sensitivity for the four cases. Grayscale goes from -0.15 times the maximum sensitivity to the maximum.

covered by the lowest few terms. For an equatorial view and a $4 \mathrm{~km} \mathrm{~s}^{-1}$ rotation rate the numbers are $66.3 \%, 94.7 \%, 98.7 \%$, and $99.8 \%$, for one through four terms. For a single $\left(4 \mathrm{~km} \mathrm{~s}^{-1}\right)$ rotation rate, but different viewing angles, the corresponding numbers are $84.6 \%, 97.4 \%, 99.5 \%$, and $99.9 \%$. For the case with different angles and rotation rates the numbers are $89.3 \%$, $97.7 \%, 99.5 \%$, and $99.8 \%$.

Given these results it appears that one only needs to fit two or three terms to the observed spectra to extract the vast majority of the available information. Exactly how many terms are needed to extract all the statistically significant information depends on the signal-to-noise ratio, resolution, and spectral coverage. The similarity of the spectra also means that there is little need to know the inclination and rotation rate in order to fit the spectra, which greatly simplifies the fitting. The analysis of the multiple resulting time series is discussed in Sect. 3.4.

The singular vectors in mode ID $(\boldsymbol{U})$ give the relative visibility of a given $\boldsymbol{V}$ to various modes, as shown in Fig. 14. Given that $V_{1}$ is very close to the wavelength derivative it is not surprising that the corresponding $\boldsymbol{U}$ vector is dominated by the cos component. Similarly the second vector is dominated by the sin component and so forth.

To further illustrate this Fig. 15 shows $\sigma_{2} U_{2, j}$ for the sin term as a function of $\sigma_{1} U_{1, j}$ for the cos term. As can be seen the ratios of the two visibilities are different for different modes, indicating that it may be possible to partially identify the modes based on this ratio. Interestingly the ratio depends mostly on the rotation rate and $(l, m)$ and less on the inclination.

The other two lines studied give similar results. The singular vectors in wavelength are different for the IR line, but the behavior of the singular vectors in ID and the singular values are similar. Considering the three lines simultaneously does not lead to a significant improvement in the ability to separate modes.
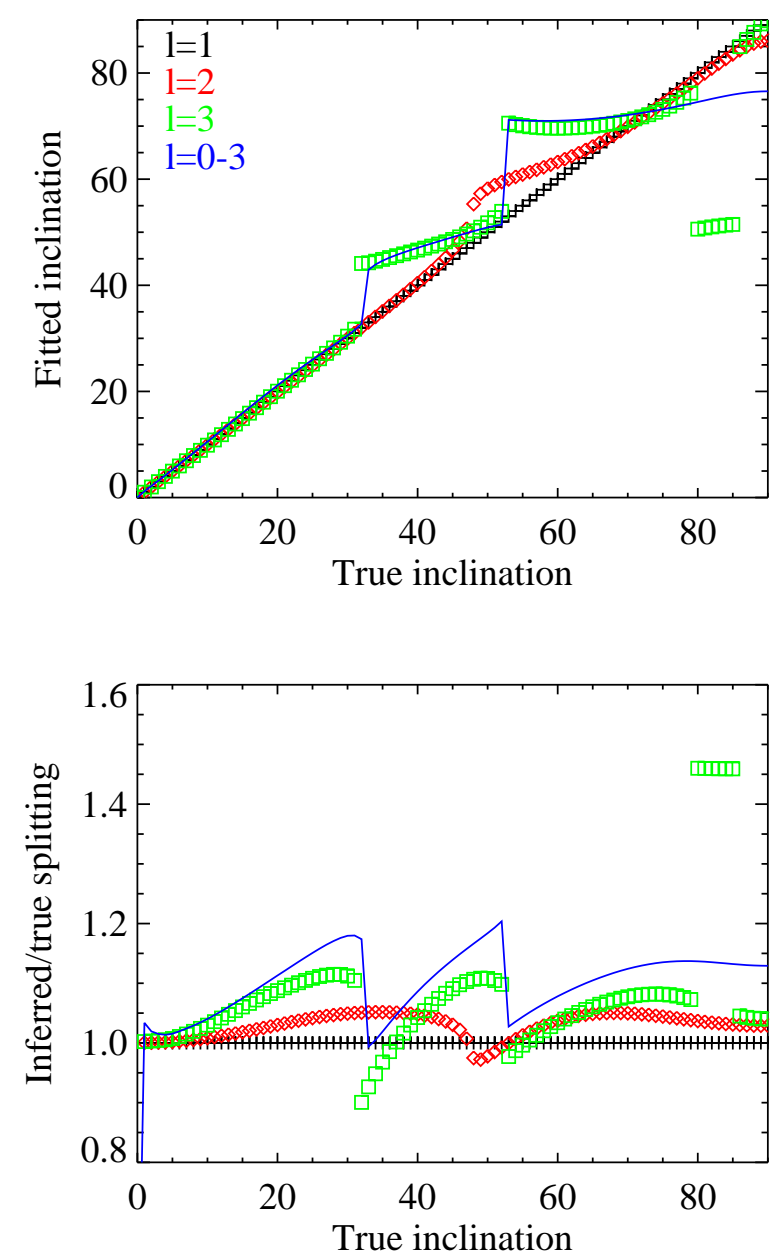

Fig. 10. Results of fitting power spectra constructed with sensitivities calculated including the effects of rotation, but using a model calculated assuming sensitivities ignoring the effects of rotation. Results are shown for the $\mathrm{G} 2$ star with no field, the $6173 \AA$ line, and a rotation velocity of $6 \mathrm{~km} \mathrm{~s}^{-1}$. The ratio of the FWHM of the modes to the rotation rate is set to 1.0. The symbols show the results of fitting the spectrum of one $l$ at a time. The solid blue line shows the results of fitting the spectra of all $l$ with $0 \leq l \leq 3$ together. In all cases a global search was performed to ensure that the global minimum has been found.

It is not practical to perform a brute-force calculation of the perturbations for the entire spectrum. The radiative transfer calculations are extremely expensive given the size of the computation boxes and the number of lines present in a typical spectrum. On the other hand the majority of lines behave in a similar way and their properties vary slowly with atomic parameters, so it is only necessary to perform a detailed radiative transfer calculation for a subset. Also, the number of spatial points needed to sample the convection adequately can likely be reduced dramatically.

Whether it is possible to determine the singular vectors in wavelength empirically (e.g., by performing an SVD of a number of observed spectra) is unclear as there are many other sources of variations, such as spectrograph drift and differential extinction. Also, such an empirical determination does not directly identify the modes.

Another way to determine the vectors without having to perform the radiative transfer would be to use the method of Dravins et al. (2017), which has the advantage of automatically taking into account the spectrograph PSF, but the obvious 


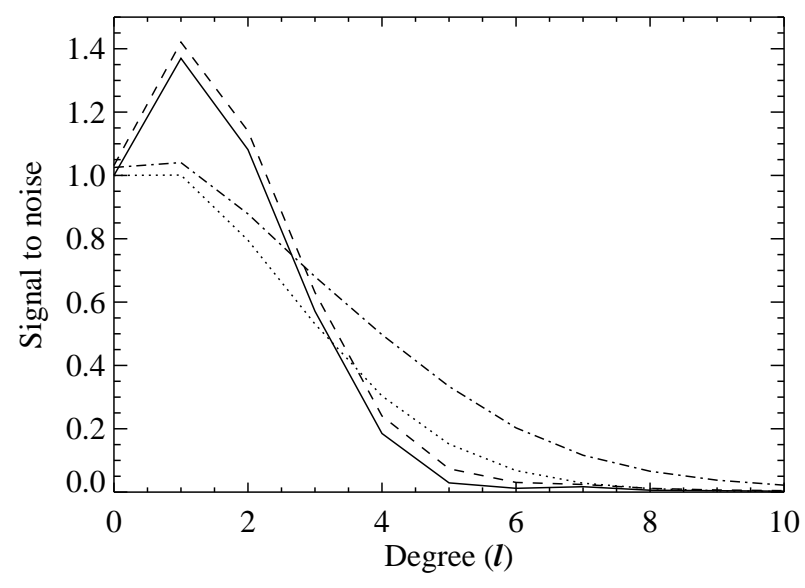

Fig. 11. Signal to noise for various cases. Solid is cross-correlation is $m=0$ for $i=0^{\circ}$ and dashed the corresponding fits. The dotted line indicates the cross-correlation for $m=l, 4 \mathrm{~km} \mathrm{~s}^{-1}$ equatorial rotation and $i=90^{\circ}$ while the dash-dotted line indicates the corresponding fit. In all cases the absolute value of the signal-to-noise ratio is shown divided by the corresponding $l=0$ cross-correlation value.

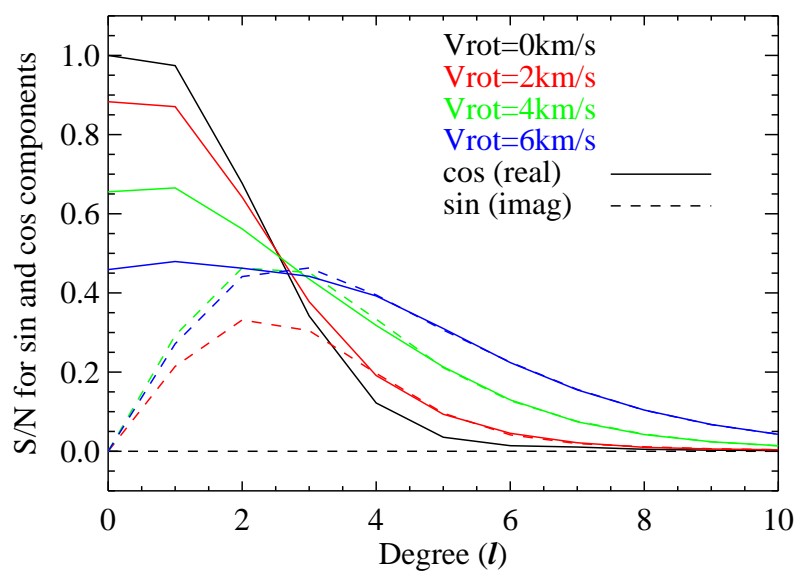

Fig. 12. Signal to noise for the real (cos) and imaginary (sin) components for $m=l, i=90^{\circ}$ for various equatorial rotation velocities. The signal-to-noise ratios were normalized to the $l=0$ case without rotation.

disadvantage is that it only works directly for stars with large transiting planets.

Similarly, the fact that the SVD represents the most compact representation does not mean that this method has to be employed. Other parameterizations, such as moments (Briquet \& Aerts 2003), can also be used, but it must be realized that more terms may be needed and/or that some information is lost.

It is possible that the addition of the thermodynamic perturbations caused by the modes or some of the other neglected effects increases the ability to discriminate between modes, but this will have to await 3D simulations capable of predicting these effects reliably.

Implementing the fits in practice will require a number of steps. Ideally this would start by obtaining an (M)HD model for the specific stellar type, synthesizing the spectrum as a function of viewing angle, convolving with the spectrograph PSF, integrating up the perturbations caused by each mode, performing the SVD, determining how many terms to retain, fitting the spectra using the relevant number of terms, and fitting the resulting time-series, as described in the next subsection. Unfortunately, many of these steps have complications.

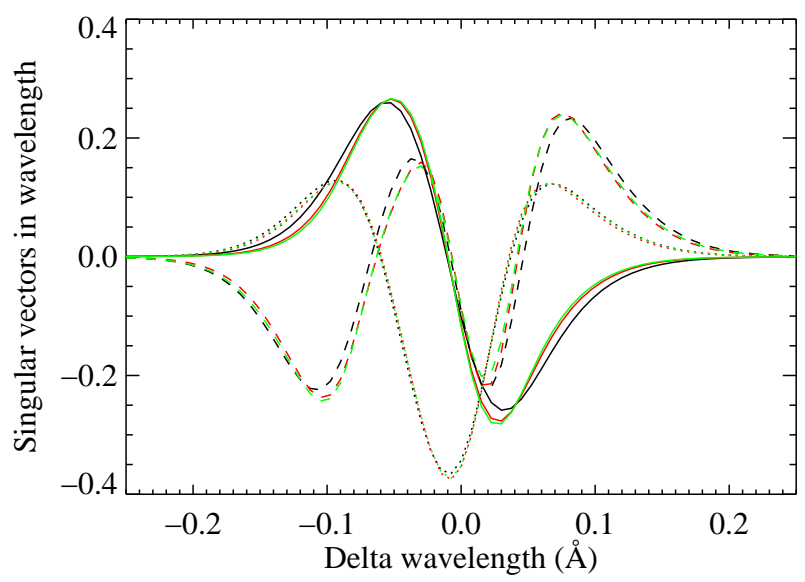

Fig. 13. Singular vectors in wavelength for three cases. Black: $i=90^{\circ}$ with $4 \mathrm{~km} \mathrm{~s}^{-1}$ equatorial rotation rate. Red: All inclinations $\left(i=0^{\circ}, 10^{\circ}, \ldots, 90^{\circ}\right)$ combined with $4 \mathrm{~km} \mathrm{~s}^{-1}$ equatorial rotation rate. Green: All inclinations combined with all equatorial rotation rates $\left(0 \mathrm{~km} \mathrm{~s}^{-1}, 1 \mathrm{~km} \mathrm{~s}^{-1}, \ldots, 6 \mathrm{~km} \mathrm{~s}^{-1}\right)$. Solid, dotted, and dashed lines indicate the first, second, and third vectors, respectively. The signs of some of the vectors were inverted; they are arbitrary in an SVD. All cases are for the $6173 \AA$ line at $0 \mathrm{G}$.
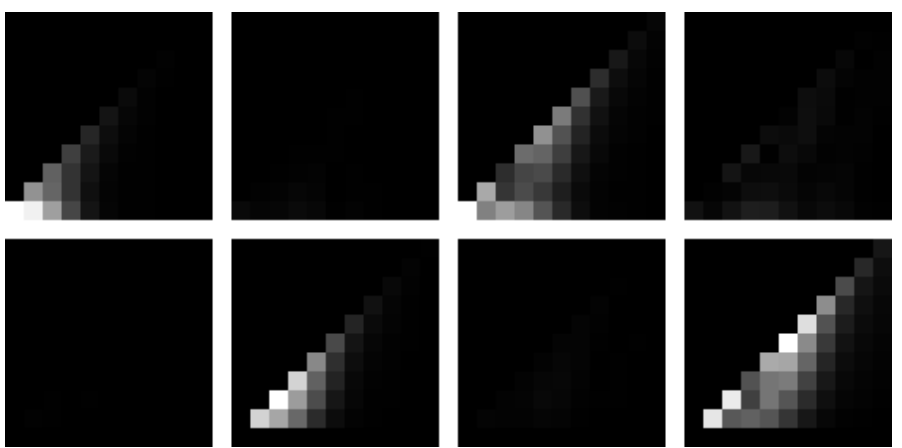

Fig. 14. First four (from left to right) singular vectors in $l, m$, and $p$ for the case shown in red in Fig. 13. Top row shows the cos component and the bottom row shows the sin component. Each block shows the mean over $i$ of the absolute value of the singular vectors as a function of $l$ (horizontal) and $m$ (vertical) for $0 \leq m \leq l \leq 10$. The blocks have been individually normalized to have identical maximum values.

The simulation and spectral synthesis are extremely costly if carried out with brute force. However, as demonstrated the resulting visibilities only depend weakly on stellar type and most of the spectral lines of interest are expected to depend only slowly on the properties of the lines, making it possible to interpolate the results. Indeed, simply interpolating the results shown in this paper, possibly supplemented with a few extra stellar models and spectral lines may be adequate, given the limited signal to noise of the currently available spectra.

The effects of the spectrograph PSF have not been discussed here. If the PSF is well known it is straightforward to convolve by it after the line synthesis and to check how the results are affected. If the PSF is poorly known, more research will obviously be needed.

Integrating up the contributions for each mode and performing the SVD should similarly not present a problem. Once the signal-to-noise ratio for the spectrograph is known, determining the number of terms to retain is also straightforward to estimate, although of course, adjacent values could be attempted to test the significance. The fitting of the individual spectra is a linear fit of 


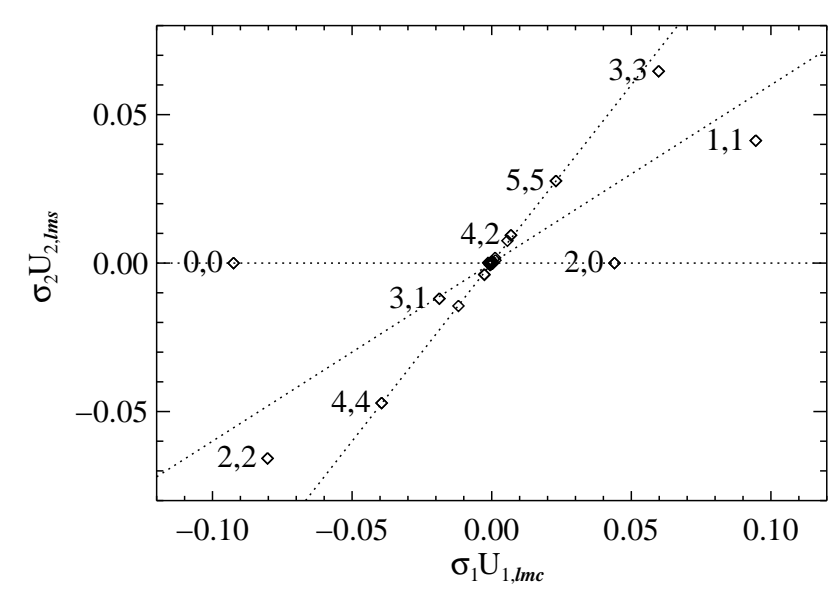

Fig. 15. Visibility of the sin component for the second term in the SVD as a function of the visibility of the cos component for the first term for the case shown with black in Fig. 13. The modes with the largest visibilities are identified by their $l, m$ values. The steepest dotted line roughly represents the largest ratio, the middle line represents half of that ratio, and the third line represents zero.

nearly orthonormal vectors and should thus be stable and easy to implement.

\subsection{Analysis of multiple simultaneous time-series}

In asteroseismology only a single time series has generally been available for a given star and analysis methods initially developed for Sun as a star observations have been used. For an introduction to the basics, see Anderson et al. (1990).

Clearly, these analysis methods will not be optimal for the multiple simultaneous time series generated using the methods described in Sects. 3.2 and 3.3. Using the least-squares analysis described in Sect. 3.2, two time series $\left(x_{\mathrm{c}}\right.$ and $\left.x_{\mathrm{s}}\right)$ are obtained, while in Sect. 3.3 there can be several time series, depending on the signal-to-noise ratio. Thus the question arises of how to go about the case where each mode appears in multiple series, but with different visibilities. This issue has been addressed extensively in helioseismology and details can be found in, for example, Schou (1992) and Larson \& Schou (2015). I briefly outline the general idea and how it might be applied to the present case. I restrict my case to stochastically driven solar-like oscillations.

In the case of stochastically driven solar-like oscillations, the Fourier transform of a single mode of oscillation has real and imaginary parts with a mean of zero and a variance given by

$V(v)=\frac{P / w}{1+\left(\frac{v-v_{0}}{w}\right)^{2}}$,

where $V$ is the variance, $v$ the frequency, $v_{0}$ the mode frequency, $P$ is proportional to the mode power, and $w$ is the HWHM of the mode. Under certain assumptions, most importantly that the mode excitations are frequent and that there are no gaps in the time series, it may be shown that the values of the Fourier transforms are normally distributed and are independent across frequencies and real/imaginary.

When a mode is observed with one of the methods described above, the result is that the resulting Fourier transform are multiplied by complex constants $C_{j k}$, where $j$ identifies the time series and $k$ encodes the mode. The real part of the constant is the sensitivity to the $\cos (m \phi)$ component and the imaginary part the sensitivity to the $\sin (m \phi)$ component, as discussed earlier. For simplicity, and consistent with the rest of the paper, it is assumed that these constants are independent of frequency and thus the radial order $n$ of the mode.

As it has been assumed that the amplitudes are small, it follows that the observed transforms $(\tilde{y})$ are the sum over the transforms $(\tilde{x})$ of the individual modes

$\tilde{y}_{j}(v)=\sum_{k} C_{j k} \tilde{x}_{k}(v)$

An important consequence of the fact that a given mode, in general, appears in more than one time series is that fitting their power spectra (as opposed to Fourier transforms) is suboptimal. Specifically, the fact that the phases are correlated between the Fourier transforms is ignored resulting in a loss of information when the power spectra are made from the Fourier transforms.

While it is possible to continue using complex numbers (e.g., through the use of cross-spectra), it is perhaps simpler and more intuitive to rearrange the equations to be purely real. To this end the complex spectra are split into twice as many real spectra to give

$\tilde{y}_{j}^{\prime}(v)=\sum_{k} C_{j k}^{\prime} \tilde{x}_{k}^{\prime}(v)$

where $j$ and $k$ now both run over the real and imaginary parts of the observed and mode transforms.

As the $\tilde{y}_{j}^{\prime}$ are sums of normally distributed variables with zero mean, they are themselves normally distributed with zero mean and a covariance matrix with elements given by

$E_{n m}\left(\boldsymbol{a}, v_{i}\right)=\sum_{k} C_{n k}^{\prime}(\boldsymbol{a}) C_{m k}^{\prime}(\boldsymbol{a}) V_{k}\left(\boldsymbol{a}, v_{i}\right)+E_{n m}^{\mathrm{noise}}\left(\boldsymbol{a}, v_{i}\right)$,

where the vector $\boldsymbol{a}$ containing the parameters describing the modes has been made explicit and where a noise term has been added. It is important to note that $E$ depends on the parameter vector $\boldsymbol{a}$ through both $C^{\prime}$ and $\boldsymbol{V}$.

The probability density for such a multivariate normal distribution is given by

$P\left(\boldsymbol{a}, v_{i}\right)=\left|2 \pi E\left(\boldsymbol{a}, v_{i}\right)\right|^{-1 / 2} \exp \left(-\frac{1}{2} y\left(v_{i}\right)^{\mathrm{T}} E\left(\boldsymbol{a}, v_{i}\right)^{-1} y\left(v_{i}\right)\right)$,

where $\|$ indicates the determinant.

To obtain the maximum-likelihood estimate it is necessary to minimize the negative of the logarithm of the product of the probabilities, that is

$S(\boldsymbol{a})=\sum_{i} \log \left|E\left(\boldsymbol{a}, v_{i}\right)\right|+y\left(v_{i}\right)^{\mathrm{T}} E\left(\boldsymbol{a}, v_{i}\right)^{-1} y\left(v_{i}\right)$,

where the sum only needs to be made over positive frequencies, given the lack of independent information in the negative part of the transforms. It is important to note that, despite the superficial similarity, this is not a least-squares fit. In a least-squares fit, one fits for the mean with a known variance, here the mean is known (zero) and the fit is for the variance. This results in a nonlinear fit, but one that is straightforward to implement from a numerical point of view using standard numerical routines. Equivalent Bayesian estimates should be straightforward to calculate.

As for implementing such a fit in practice, several things need to be considered. First of all I have not explicitly stated the exact list of parameters in the parameter vector $\boldsymbol{a}$ used to describe the modes, as a large variety of parameterizations can and have been used, depending on the star and preferences of various 
investigators. Having said that, one would expect that parameterizations similar to those in current use (e.g., Nielsen et al. 2014) should work here, which some parameters added to deal with the noise covariance.

Similarly, I have not specified how the noise term could be parameterized, but likely the form of the covariance matrix can be determined from data away from the peaks, as done in helioseismology, given that it would be expected to depend slowly on frequency.

It may be noted that a brute force implementation of the above is actually somewhat inefficient. In particular many of the coefficients in $C^{\prime}$ may be almost purely real or imaginary and the covariance matrix $E$ has many identical entries and zeros. However, given that that there are only a few time series, the time saved for a maximum-likelihood estimate by a more efficient computation is unlikely to be worth the effort. For an MCMC calculation this may not be the case.

\section{Discussion}

The analysis described in this paper is, of course, incomplete and many effects have been neglected. For example the stars have been assumed to have no differential rotation in latitude, the distortion of the eigenfunctions by rotation has been neglected, the displacement has been assumed to be purely radial and independent of height, and it has been assumed that there are no thermodynamic perturbations accompanying the oscillations. For a discussion of some of these effects see Zima (2006). The assumptions of height independence and lack of thermodynamic perturbations are difficult to address numerically, as the signalto-noise ratio of the oscillations in the MHD simulations is very small because of their limited size and short simulation time. For the Sun there is a noticeable height dependent effect (Zhao et al. 2012) in the observations and Baldner \& Schou (2012) have shown that the height dependence in the simulations is far from that predicted by simple theoretical models. The thermodynamic perturbations are difficult to address by the MHD models due to the signal-to-noise limitations, but given the discrepant visibilities reported by Lund et al. (2014) in intensity and the complexity of the physics it should not be assumed that the simple analytical models often used give reliable results. However, for slowly rotating Sun-like stars the effects considered almost certainly dominate over those neglected, at least for spectroscopic observations.

For photometric observations the situation is different. The present method predicts essentially no perturbations and to obtain reliable estimates the details of the thermodynamic perturbations with height have to be understood in detail, as discussed above. It should also be mentioned that the issues discussed in this paper are also highly relevant for Doppler imaging and that the two areas have many similarities.

\section{Conclusions}

In summary it is clear that significant improvements can be made by careful modeling and analysis of the data and that while some of the changes are modest they should nonetheless be taken into account in the analysis of stellar spectra. Having said that, it is also clear that future work is needed in order to understand some of the remaining uncertainties and it would be useful to determine if the observed mode visibilities agree with those predicted in this work.

Acknowledgements. I would like to thank Benjamin Beeck, Regner Trampedach, Martin Bo Nielsen, and Björn Löptien for useful discussions and help with various calculations. The HMI data used are courtesy of NASA/SDO and the HMI science team.

\section{References}

Anderson, E. R., Duvall, Jr., T. L., \& Jefferies, S. M. 1990, ApJ, 364, 699

Auvergne, M., Bodin, P., Boisnard, L., et al. 2009, A\&A, 506, 411

Baldner, C. S., \& Schou, J. 2012, ApJ, 760, L1

Bazot, M., Bouchy, F., Kjeldsen, H., et al. 2007, A\&A, 470, 295

Bedding, T. R. 2014, in Asteroseismology, eds. P. L. Pallé, \& C. Esteban (Cambridge, UK: Cambridge University Press), 60

Beeck, B., Cameron, R. H., Reiners, A., \& Schüssler, M. 2013, A\&A, 558, A48 Borucki, W. J., Koch, D., Basri, G., et al. 2010, Science, 327, 977

Bouchy, F., Pepe, F., \& Queloz, D. 2001, A\&A, 374, 733

Briquet, M., \& Aerts, C. 2003, A\&A, 398, 687

Brown, T. M., Kotak, R., Horner, S. D., et al. 1998, ApJS, 117, 563

Chaplin, W. J., Basu, S., Huber, D., et al. 2014, ApJS, 210, 1

Dravins, D., Ludwig, H.-G., Dahlén, E., \& Pazira, H. 2017, A\&A, 605, A91

Fletcher, S. T., Chaplin, W. J., Elsworth, Y., Schou, J., \& Buzasi, D. 2006 MNRAS, 371, 935

Frutiger, C., Solanki, S. K., Fligge, M., \& Bruls, J. H. M. J. 2000, A\&A, 358, 1109

Gizon, L., \& Solanki, S. K. 2003, ApJ, 589, 1009

Grundahl, F., Christensen-Dalsgaard, J., Arentoft, T., et al. 2009, Commun. Asteroseismol., 158, 345

Grundahl, F., Fredslund Andersen, M., Christensen-Dalsgaard, J., et al. 2017, ApJ, 836, 142

Larson, T. P., \& Schou, J. 2015, Sol. Phys., 290, 3221

Lund, M. N., Kjeldsen, H., Christensen-Dalsgaard, J., Handberg, R., \& Silva Aguirre, V. 2014, ApJ, 782, 2

Lund, M. N., Silva Aguirre, V., Davies, G. R., et al. 2017a, ApJ, 835, 172

Lund, M. N., Silva Aguirre, V., Davies, G. R., et al. 2017b, ApJ, 850, 110

Nielsen, M. B., Gizon, L., Schunker, H., \& Schou, J. 2014, A\&A, 568, L12

Pereira, T. M. D., Asplund, M., Collet, R., et al. 2013, A\&A, 554, A118

Rauer, H., Catala, C., Aerts, C., et al. 2014, Exp. Astron., 38, 249

Ricker, G. R., Winn, J. N., Vanderspek, R., et al. 2014, in Space Telescopes and Instrumentation 2014: Optical, Infrared, and Millimeter Wave, Proc. SPIE, 9143, 914320

Schou, J. 1992, PhD Thesis, Aarhus University, Aarhus, Denmark

Schou, J. 2014, in Precision Asteroseismology, eds. J. A. Guzik, W. J. Chaplin, G. Handler, \& A. Pigulski, IAU Symp., 301, 481

Schou, J., Scherrer, P. H., Bush, R. I., et al. 2012, Sol. Phys., 275, 229

Stello, D., Huber, D., Bedding, T. R., et al. 2013, ApJ, 765, L41

Walker, G., Matthews, J., Kuschnig, R., et al. 2003, PASP, 115, 1023

Zhao, J., Nagashima, K., Bogart, R. S., Kosovichev, A. G., \& Duvall, Jr., T. L. 2012, ApJ, 749, L5

Zima, W. 2006, A\&A, 455, 227 